\title{
A cost-based importance measure for system components: an extension of the Birnbaum importance
}

\author{
Shaomin $\mathrm{Wu}^{1}$ \\ Kent Business School, University of Kent, \\ Canterbury, Kent CT2 7PE, United Kingdom \\ Frank P.A. Coolen \\ Department of Mathematical Sciences, Durham University, \\ Durham DH1 3LE, UK
}

\begin{abstract}
In reliability engineering, component importance measures are used to prioritise components in a system for purposes such as reliability improvement and maintenance planning. Existing importance measures have paid little attention to the costs incurred by maintaining a system and its components within a given time period. Cost-effectiveness analysis, however, is critically important in increasingly competitive markets. This paper proposes a new cost-based importance measure which considers costs incurred by maintaining a system and its components within a finite time horizon. Possible extensions are discussed and examples are given to show the use of the new measure.
\end{abstract}

ऋ Recommended citation: Wu, S., Coolen, F.A. (2013) A cost-based importance measure for system components: an extension of the Birnbaum importance, European Journal of Operational Research, 225, pp. 189-195 
Keywords: Birnbaum importance, structural function, cost-based importance, BarlowProschan importance, risk-based importance.

\section{Introduction}

In reliability engineering, component importance measures are used to prioritise components in a system for purposes such as reliability improvement and maintenance planning. Existing importance measures, however, have paid little attention to the costs incurred by maintaining binary systems and their components within a given time period. This issue is discussed in this paper.

\subsection{Prior work}

Component importance has been studied by many authors $[1,2,3,4,5,6,7,8,9,10,11,12,13,14,15,16]$. Well-known importance measures include Birnbaum importance [1], BarlowProschan importance [2], Fussell-Vesely importance [3], Natvig importance [4], importance for multistate systems [5,7,8], structure importance [6,8] and joint importance [89]. There is a substantial literature on such importance measures, discussing specific theoretic aspects and their practical use, e.g. [9,14,15]. When interpreting component importance, Rausand and Hoyland concluded that the importance of a component should depend on the following factors[17]:

- the location of the component in the system;

- the reliability of the component;

- the uncertainty in the estimate of the component reliability and related cost;

In this paper, we argue that the importance of a component should, in addition to these factors, also depend on the costs of maintaining this component in

$\overline{1}$ Corresponding author. Email: s.m.wu@kent.ac.uk. Telephone: 00441227827940. 
a given time interval $(0, t)$, and we propose a new importance measure that takes these costs into account.

\subsection{Problems}

We consider the consequences caused by improving the reliability of a component within a time interval $(0, t)$ by considering the following three types of costs, which we refer to later as Cost1, Cost2 and Cost3.

Cost1: Costs of improving component reliability. In a system, the costs of improving the reliabilities of different components are likely to be different, although the levels of improvement might be the same. In a drinking water supply system, for example, if one wants to increase the same amount of reliability on different components, the costs of improving the reliability of a water pump are typically different to the costs of improving the reliability of a switch board.

Cost2: Costs due to component failure. If a component fails, it needs to be repaired or replaced. This incurs costs. For example, in a drinking water system, a failed pumping station needs to be repaired. Such costs typically vary for different components and therefore should be distinguished in an importance measure.

Cost3: Cost of system failure. A system is usually designed and installed for completing a specific function. If a system fails, it can cause losses such as loss of lives, damage to health, release of hazardous materials or other detrimental effects to the environment, or economic losses including repair or replacement of the directly damaged structure as well as repair of collateral damage. For example, if a water supply system fails, its users might need to stop their production lines. This can cause economic losses, which can include losses to the supplier due to possible penalties relating to interruption of water supply and the effects on their reputation. 
Cost1 has been addressed in several publications, e.g. [17, p.188]. Little research, however, has considered Cost2 and Cost3 in reliability importance measures for binary systems, although performance utility has been incorporated in importance measures for multistate systems $[5,7,8$. However, when selecting components to improve their reliabilities in order to improve the reliability of the entire system, one must consider the different costs incurred, as the ultimate purposes of improving the reliability of a system are to prolong the system's service life and to save on costs of maintaining the system. This necessitates the introduction of a new importance measure that reflects Cost2 and Cost3 and distinguishes between them.

This paper introduces a new importance measure that considers and distinguishes the cost of system failures (Cost2) from the cost of component failures (Cost3). Section 2 introduces assumptions and notation. Section 3 introduces a new cost-based importance measure, derives its properties and considers it for some specific systems. In Section 4 some further aspects of this measure are discussed. Section 5 presents a numerical example and a small illustrative case study of the use of the cost-based importance. Section 6 concludes the paper.

\section{Assumptions and Notation}

In this paper, we make the following assumptions.

A.1 A system is composed of $n$ components. At time $t=0$, all components are new.

A.2 The components and the system are binary, i.e., having two possible states: functioning or failed.

A.3 All components in the system are repairable. The quality of each repair upon failure is minimal. That is, repairs upon failed components are minimal and repair upon system failures is also minimal. A minimal repair restores the item to the state it had just before failure. 
A.4 The system is coherent, that is, the failure of one or more components cannot lead to improved functioning of the system.

A.5 The behaviours of the components in the system are mutually statistically independent.

A.6 When a component is waiting for repair, it neither ages nor deteriorates. Thus, it does not fail.

A.7 Compared to the operating time, repair times are negligible.

Let $X_{k}(t)$ be a binary random variable representing the state of component $k$ at time $t \geq 0$, for $k=1,2, \ldots, n$. The random variable $X_{k}(t)=1$ if the component is functioning at $t$ and $X_{k}(t)=0$ otherwise. We assume that the stochastic process $X_{k}(t), t \geq 0$, has right-continuous sample paths. The assumption of the system being coherent implies that the structure function $\phi(\mathbf{X})$ of the system, where $\mathbf{X}(t)=\left(X_{1}(t), X_{2}(t), \ldots, X_{n}(t)\right)$, is non-decreasing in each argument and we further assume that $\phi(\mathbf{0})=0$ and $\phi(\mathbf{1})=1$, which excludes only trivial systems that either never or always function independent of the state of the components. The components are assumed to be independent, i.e. the processes $X_{k}(t), k=1,2, \ldots, n$, are stochastically independent. To summarise, the system is a monotone binary system of $n$ independent components.

Table 1 presents further notation used in this paper.

\section{Here: Table 1}

\section{Cost-based component importance}

Birnbaum introduced the following measure of the reliability importance of a component in a system [1]:

Definition 1 The Birnbaum reliability importance (BRI) is defined by [1]

$$
I_{k}^{B}(t)=\frac{\partial R_{s}(t)}{\partial R_{k}(t)}
$$


where $k=1,2, \ldots, n$. The Birnbaum importance can be interpreted as follows: if $I_{k}^{B}(t)$ is large, a small change in the reliability of component $k$ will result in a comparatively large change in the system reliability.

As discussed in Section 1.2, the total cost of maintaining a system within time period $(0, t)$ includes costs due to system failure and maintaining failed components. Hence, the total maintenance cost within time interval $(0, t)$ is given by

$$
C(t)=\sum_{k=1}^{n}\left(C_{s, k}(t)+C_{k}(t)\right)
$$

where $C_{s, k}(t)$ is the expected total cost of maintaining the system within time $(0, t)$, caused by the failure of component $k$, and $C_{k}(t)$ is the expected total cost of maintaining component $k$ within time $(0, t)$. We distinguish between the expected total costs of maintaining the system caused by different components, in order to deal with the fact that system production loss relates to the maintenance times for failed components, which typically differ per type of component (e.g. [18]).

We develop the two elements in the right-hand side of Eq. (2) further for two specific scenarios, to illustrate the new approach presented in this paper. Application of the new cost-based importance measure to real-world problems will typically be more complex, relevant issues are discussed later in this paper leading to interesting and challenging topics for further research.

Scenario A. Once a component fails, it can immediately be detected and repaired.

Scenario B. We make the following assumptions.

B1. The failure of a component may not be detected immediately, but it will be inspected and repaired once the system fails. That is, a failed component will be repaired once a minimal cut set containing this component fails.

B2. Once a minimal cut set fails, only the components in this failed minimal cut set are repaired. Failed components that are not contained in this minimal cut set will not be repaired. 
When choosing a component for improvement, we can aim to minimise the value of $C(t)$, or to maximise the value of $-C(t)$. This leads to the following definition.

Definition 2 The cost-based importance of component $k$ is defined as

$$
I_{k}^{C}(t)=-\frac{\partial C(t)}{\partial R_{k}(t)}
$$

The importance measure $I_{k}^{C}(t)$ can be interpreted as follows: when $I_{k}^{C}(t)$ is large, a small change in the reliability of component $k$ will result in a comparatively large change in the total cost of maintaining the entire system during time interval $(0, t)$.

While $I_{k}^{B}(t)$ was introduced for non-repairable systems, $I_{k}^{C}(t)$ can be used for repairable systems and components when minimal repairs upon failures are conducted. The reason can be explained as follows. Take component $k$ as an example. The system starts at time 0 . The first failure of component $k$ is governed by the distribution $F_{k}(t)$ with failure rate $r_{k}(t)$, and each succeeding failure is governed by the intensity function $\lambda_{k}(t)$. When repair upon failure is minimal, the failure intensity $\lambda_{k}(t)$ has the same functional form as the failure rate governing the first system failure. That is, $\lambda_{k}(t)=r_{k}(t)$. Since, mathematically, $R_{k}(t)=e^{-\Lambda_{k}(t)}\left(\right.$ where $\Lambda_{k}(t)=\int_{0}^{t} \lambda(x)$ x $)$, we can use $I_{k}^{C}(t)$ to measure the importance of component $k$ in a repairable system.

Since $\Lambda_{k}(t)=-\ln \left(R_{k}(t)\right)$, the definition of the importance in Eq. (3) can be re-written as

$$
\begin{aligned}
I_{k}^{C}(t) & =-\frac{\partial C(t)}{\partial R_{k}(t)} \\
& =-\frac{\partial C(t)}{\partial \Lambda_{k}(t)} \frac{\partial \Lambda_{k}(t)}{\partial R_{k}(t)} \\
& =\frac{1}{R_{k}(t)} \frac{\partial C(t)}{\partial \Lambda_{k}(t)}
\end{aligned}
$$




\subsection{Cost-based importance for Scenario $A$ and Scenario $B$}

Assume a system is composed of three components: 1, 2, and 3, as shown in Figure 1.

\section{Here: Figure 1}

In the following, we investigate the proposed importance measure under Scenario A and Scenario B, respectively.

\subsubsection{Scenario A}

Take the system shown in Figure 1 as an example, component 1 constitutes a first-order cut set: the system fails if component 1 fails. Components 2 and 3 constitute another minimal cut set. Under Scenario A, if one of the components 2 and 3 fails, it will be repaired immediately and hence the system will not fail. As such, the failure of component 2 or component 3 only incurs cost of repairing the component itself.

Now let's look at more general situations. Under Scenario A, a failed component will be repaired immediately. Hence, the system will fail only if the failure of a component, component $i_{j}$ say, can cause system failure, and then incur cost $c_{s, i_{j}}$ of system failure as well as the cost of repairing component $i_{j}$ itself. If the failure of a component does not cause system failure, it will only incur cost of repairing the failure component.

Thus, the total expected maintenance cost within time interval $(0, t)$ is given by

$$
C(t)=\sum_{j=1}^{m_{0}} c_{s, i_{j}} \Lambda_{U_{i_{j}}}(t)+\sum_{k=1}^{n} c_{k} \Lambda_{k}(t)
$$

where $c_{s, i_{j}}$ is the cost per system failure caused by failure of component $i_{j}$ and $c_{k}$ is the cost of failure of component $k . \Lambda_{U_{i_{j}}}$ is the expected number of failures of minimal cut set ii $U_{i_{j}}$ that is composed of one component, component $i_{j}$,

$\overline{\text { ii }}$ A state vector $\mathbf{x}$ is called a cut vector if $\phi(\mathbf{x})=0$. If $\phi(\mathbf{y})=1$ for all $\mathbf{y}>\mathbf{x}$, 
i.e., $U_{i_{j}}$ is a first-order cut set.

Thus, we have: under Scenario A, if component $k$ constitutes a first-order cut set, then $I_{k}^{C}(t)=\left(c_{k}+c_{s, k}\right) / R_{k}(t)$; if component $k$ is not in a first-order cut set, then $I_{k}^{C}(t)=c_{k} / R_{k}(t)$.

\subsubsection{Scenario B}

Generally, under Scenario B, a system fails and then is repaired only if at least one minimal cut set failed. Assume that there are $n_{0}$ minimal cut sets in the system, then the cost incurred within time interval $(0, t)$ is composed of two parts: one on cost of repairing the system upon failures of the $n_{0}$ minimal cut sets, and one on cost of repairing each component in the failed minimal cut sets. That is, the expected cost of maintaining the system within time interval $(0, t)$ is given by

$$
C(t)=\sum_{i=1}^{n_{0}}\left(\Lambda_{U_{i_{1}, \ldots, i_{m_{i}}}}(t)\left(c_{c, i}+\sum_{j=1}^{m_{i}} c_{i_{j}}\right)\right)
$$

where $c_{c, i}$ is the expected cost per system failure, caused by the failure of the minimal cut set $U_{i_{1}, \ldots, i_{m_{i}}}$, and $c_{i_{j}}$ is the expected cost per failure of component $i_{j}$.

Eq. (6) implies: if components $k_{1}$ and $k_{2}$ are only included in the minimal cut set $U_{i_{1}, \ldots, i_{m_{i}}}$ but not included in any other minimal cut sets, then the values of $c_{k_{1}}$ and $c_{k_{2}}$ do not impact the ordering of $I_{k_{1}}^{C}$ and $I_{k_{2}}^{C}$.

Estimating $\Lambda_{U_{i_{1}, \ldots, i_{m_{i}}}}(t)$ in Eq. (6) is more complicating. Similar to the analysis in Scenario A, we take the system shown in Figure 1 as an example. Without loss of generality, assume components 1,2 , and 3 fails at times $t_{1}, t_{2}$, and $t_{3}$, successively, as shown in Figure 2.

\section{Here: Figure 2}

then $\mathrm{x}$ is a minimal cut vector. If $U_{i_{1}, \ldots, i_{m_{i}}}$ is a minimal cut vector, then the set $U_{i_{1}, \ldots, i_{m_{i}}}=\left\{i_{j}: x_{i_{j}}=0\right.$ and $\left.j=1, \ldots, m\right\}$ is a minimal cut set. 
We have the following analysis.

- At time $t_{1}$, component 1 fails, which causes the system fail. Component 1 is repaired and then the system starts again. As the repair is minimal and the repair time is negligible, the failure process of component 1 can be seen as a non-homogeneous Poisson process (NHPP), for example. Thus, it can be easy to estimate the number of failures and cost of those failures within a given time period $(0, t)$ for component 1 , that is, estimating $C_{1}(t)$ is not problematic.

- At time $t_{2}$, component 2 fails, which does not cause the system fail. According to Assumption B1 of Scenario B, component 2 will not be repaired until time $t_{3}$ when the system fails due to the failure of component 3 . That is, at time $t_{3}$, components 2 and 3 are repaired and then the system is re-started again. However, as the failure of component 2 is not detected immediately after it failed, time $t_{2}$ is unknown. Hence, the actual working time of component 2 within time interval $(0, t)$ (with $\left.t>t_{3}\right)$ is not obtainable. Therefore, an explicit expression of $C_{2}(t)$ might not easily be obtained. Similar analysis applies to component 3 .

\subsubsection{A special case of Scenario B}

Now let's look at a special case that satisfies the following two more conditions.

B3. All of the minimal cut sets in the system are mutually exclusive, that is, a component in the system only belongs to one minimal cut set;

B4. Assume the lifetimes of all of the components in the system are exponentially distributed, with intensities $\theta_{k}$ (with $\left.k=1,2, \ldots, n\right)$, respectively. That is, the lifetime distribution function of component $k$ is given by $F_{k}(t)=1-e^{-\theta_{k} t}$.

Assumption B3 ensures that the number of failures of the system is the sum of the numbers of failures of each minimal cut sets. 
Based on Assumption B4, it is know that the exponential distribution has the memorylessness property. For example, component 2 in Figure 2 in time interval $\left(t_{3}, \infty\right)$ has the same lifetime distribution $F_{2}(t)$ as that in $\left(0, t_{2}\right)$, due to

$$
\operatorname{Pr}\left(T>t+t_{2} \mid T>t_{2}\right)=\operatorname{Pr}(T>t) .
$$

Eq. (7) implies that component 2 is renewed at time $t_{3}$. Similarly, component 3 is renewed at time $t_{3}$. That is, the minimal cut set constituted with components 2 and 3 are renewed at time $t_{3}$. To be more general, the failure of the minimal cut set $U_{i_{1}, \ldots, i_{m_{i}}}$ occurs only if all of the components $i_{1}, \ldots, i_{m_{i}}$ have failed. As such, the lifetime distribution of $U_{i_{1}, \ldots, i_{m_{i}}}$ is given by $G_{U_{i_{1}, \ldots, i_{i}}}(t)=\prod_{k \in\left\{i_{1}, \ldots, i_{m_{i}}\right\}} F_{k}(t)$. As each component in $U_{i_{1}, \ldots, i_{m_{i}}}$ is renewed, the total number of renewals in time interval $(0, t)$ is given by

$$
\Lambda_{U_{i_{1}, \ldots, i_{m_{i}}}}(t)=G_{U_{i_{1}, \ldots, i_{m_{i}}}}(t)+\int_{0}^{t} \Lambda_{U_{i_{1}, \ldots, i_{m_{i}}}}(t-s) \mathrm{G}_{U_{i_{1}, \ldots, i_{m_{i}}}}(s) .
$$

The above equation is the renewal function and its derivation process can be found from textbooks of stochastic processes.

\subsection{Series and parallel systems}

For series and parallel systems, the cost-based importance for Scenario A and Scenario B are given in the following lemmas. The proofs are easily obtained.

Lemma 1 Under Scenario A or Scenario B, the total maintenance cost of a series system is given by

$$
C(t)=\sum_{k=1}^{n} \Lambda_{k}(t)\left(c_{s, k}+c_{k}\right)
$$

Hence, the importance of component $k$ in a series system is given by

$$
I_{k}^{C}(t)=\frac{c_{s, k}+c_{k}}{R_{k}(t)}
$$


Lemma 1 implies that if $c_{k}=c_{j}$ for $k, j=1,2, \ldots, n$, then $I_{k}^{C}(t)$ is equivalent to $I_{k}^{B}(t)$ for series systems.

Lemma 2 Under Scenario A, the total maintenance cost of a parallel system is given by

$$
C(t)=\sum_{k=1}^{n} c_{k} \Lambda_{k}(t)
$$

Hence, the importance of component $k$ in a parallel system, under Scenario $A$, is given by

$$
I_{k}^{C}(t)=\frac{c_{k}}{R_{k}(t)}
$$

Under Scenario A, a parallel system with multiple components will never fail, as a failed component can be repaired immediately after it fails, and then put back into service. Hence, a component with higher repair cost and smaller reliability is more important.

Lemma 3 Under Scenario B, the total maintenance cost of a parallel system is given by

$$
C(t)=\left(c_{s, 1}+\sum_{k=1}^{n} c_{k}\right) \Lambda_{s}(t)
$$

Here, without loss of generality, we assume that the cost of repairing the failure of the system is $c_{s, 1}$. Hence, the importance of component $k$ in a parallel system, under Scenario B, is given by

$$
I_{k}^{C}(t)=-\left(c_{s, 1}+\sum_{k=1}^{n} c_{k}\right) \frac{\partial \Lambda_{s}(t)}{\partial R_{k}(t)}
$$

Eq. (14) can also be written as

$$
\begin{aligned}
I_{k}^{C}(t) & =\frac{1}{R_{s}(t)} \frac{\partial R_{s}(t)}{\partial R_{k}(t)}\left(c_{s, 1}+\sum_{k=1}^{n} c_{k}\right) \\
& =\frac{1}{R_{s}(t)} I_{k}^{B}(t)\left(c_{s, 1}+\sum_{k=1}^{n} c_{k}\right)
\end{aligned}
$$

From Eq. 15), under Scenario B, the ordering of the components according to the cost-based importance measure is equivalent to their ordering according 
to the Birnbaum importance measure.

\section{Discussion}

In this section we discuss a range of topics that are important for application of the new cost-based importance measure proposed in this paper, and which therefore include interesting research challenges.

\subsection{Other systems}

In the preceding sections, we assumed that all components in the system under consideration are repairable. If there are non-repairable components, one needs to estimate the number of replacements instead of the number of repairs. In this case, cost of replacement should be considered. The definition in Eq. (3) is still valid, but the definition shown in Eq. (4) should be updated accordingly.

\subsection{Design cost}

Similar to the discussion by Rausand and Hoyland [17, p.188], where importanceto-cost is considered for the Birnbaum importance, to improve the system reliability we may want to change the parameter $\Lambda_{k}(t)$ by buying a higher quality component or adding preventive maintenance. Assume that we are able to determine the cost of the improvement as a function of $\Lambda_{k}(t)$, that is, $c_{d, k}(t)=c_{d, k}\left(\Lambda_{k}(t)\right)$, and that this function is strictly increasing or decreasing such that we can find its inverse function. The effect of an extra investment related to component $k$ may now be measured by 


$$
\begin{aligned}
\frac{\partial C(t)}{\partial c_{d, k}(t)} & =\frac{\partial C(t)}{\partial \Lambda_{k}(t)} \frac{\partial \Lambda_{k}(t)}{\partial c_{d, k}(t)} \\
& =I_{k}^{C}(t) R_{k}(t) \frac{\partial \Lambda_{k}(t)}{\partial c_{d, k}(t)}
\end{aligned}
$$

\subsection{Lifetime distributions, maintenance, cost, and applications}

Lifetime distributions in Scenario B. The assumption of exponential lifetime distributions in Scenario B is important, as shown in Figure 1 and its interpretation. However, if the lifetime distributions are not exponential or failed components are not repaired immediately after the system fails, the waiting time of a component (for example, $t_{4}-t_{1}$ for component 3 or $t_{4}-t_{3}$ for component 1 in Figure 2 is random and the age at which the component will re-start is random. Estimating the time that a component has been working within a time interval $(0, t)$ becomes complicated, which makes it hard to derive an explicit expression of the cost $C(t)$. In this case, a simulation study can be performed for further investigations, or approximations may be possible, which is an important topic for future research.

Maintenance policy. In practice, the cost-based importance may be dependent on the maintenance policy. For example, Scenario A assumes that a failed component will be repaired immediately upon its failure whereas Scenario B assumes that a failed component may need to wait for repair until the failure of the minimal cut set containing this component. In other words, these two scenarios involve different maintenance policies. In practice, there may be other scenarios. For example, a failed component may be detected and repaired with a probability $p$; further investigations are left for future research and are best done in direct relation to real-world applications.

Maintenance quality. In the preceding sections, we assumed that minimal repair is conducted on failed items, including both the system and the components in the system. Apparently, other levels of maintenance quality can also be considered. The quality of maintenance can e.g. be better-thanperfect, perfect, minimal, imperfect, and worse-than-before failure, which 
can lead to different types of total cost within a time interval $(0, t)$. This can lead to different versions (or extensions) of the cost-based importance measure.

Repair time. We also assumed that repair times are negligible. This assumption can obviously be relaxed. However, the expression of $C(t)$ will become more complex. Because the main purpose of this article is to introduce the cost-based importance measure we do not consider this further but it is an important topic for practical application of the cost-based importance measure.

Time-independent cost. In this paper, we assumed that $\operatorname{costs} c_{s, k}$ and $c_{k}$ are time independent. In practice, $c_{s, k}$ and $c_{k}$ may change with time, again the theory for dealing with this is best developed in line with real-world applications.

Finite time period. The cost-based importance measure introduced in this paper considers the process explicitly over a finite period of length $t$. The choice of $t$ may often follow directly from practical interest, but it is important in practical applications to study the behaviour of the cost-based importance measure as function of $t$. This use of a finite period of interest is different from many traditional approaches to cost aspects for repairable systems, which are often based on renewal theory and implicitly consider an infinite period. Recently, there has been increasing interest in aspects of reliability over finite periods, for example single cycles between inspections or replacements, which has shown that the criterion and period used can have substantial impact on the conclusions [19,20].

Applications of the cost-based importance. There will be many possible applications of the proposed importance measure. For large complex systems, repair of failures of sub-systems may often be regarded as minimal. When maintenance policy and maintenance quality are assessed, it is not hard to obtain the cost-based importance. For example, at both design stage and operation stage, or for the purpose of lifecycle costing, an optimal maintenance policy should be designed and the importance of each component should be assessed. Traditional importance measure such 
as the Birnbaum importance cannot be applied in the scenario when costs of maintaining different components are different. The proposed cost-based importance measure is designed can for this purpose. In this new importance measure, time $t$ can, for example, be the design life if the system user is interested in component importance and wishes to assess the lifecycle cost of the system, or the contract period for a maintenance agent who understands component importance and wishes to assess the contract cost.

\subsection{Other extensions}

Risk-based measures. Although we call $I_{k}^{C}(t)$ a cost-based importance measure, $C(t), C_{s, k}(t)$ and $C_{k}(t)$ in Eq. (2) can also be regarded as other consequences such as loss of lives, damage to health, releasing hazardous materials, etc. Broadly speaking, $I_{k}^{C}(t)$ can be regarded as a risk-based importance measure, as risk can be defined as involving "both uncertainty and some kind of loss or damage" [21] or as "the probability per unit time of the occurrence of a unit cost burden" 222].

An alternative cost-based importance measure. As an alternative to the measure proposed in this paper, one could define a cost-based importance measure based on a ratio such as $I_{k}^{C^{\prime}}=\frac{C_{k}(t)}{C_{s, k}(t)+\sum_{k=1}^{n} C_{k}(t)}$. Other ratio-based importance measures have been used by some authors for repairable systems [23]. $I_{k}^{C^{\prime}}$ can be interpreted as the ratio of the cost incurred by the failures of component $k$ within time $(0, t)$ to the cost of the entire system, which is different from the meaning of $I_{k}^{C}(t)$, as defined in Eq. (3).

Cost-based improvement potential. There are many other importance measures that can be extended based on the cost measure shown in Eq. (2). Here, we will not exhaust each possible extension, but we take an importance measure, improvement potential, as an example. Improvement potential is the difference between the system reliability with a perfect component $k$, and the system reliability with the actual component $k$. It can be expressed as 17 .

$$
I_{k}^{I P}(t)=I_{k}^{B}(t)\left(1-R_{k}(t)\right)
$$


One might extend the above measure to be the following cost-based improvement potential.

$$
I_{k}^{I P C}(t)=C\left(1_{k}, \mathbf{X}(t)\right)-C(\mathbf{X}(t))
$$

where $C\left(1_{k}, \mathbf{X}(t)\right)$ stands for the cost of maintaining the system when the reliability of component $k$ is improved to be 1 and the reliabilities of the other components remain the same as the original ones, and $C(\mathbf{X}(t))$ means the cost of maintaining the system when the reliabilities of the other components remain the same as the original ones, i.e. the cost $C(t)$ shown in Eq. (2). The new improvement potential, defined in Eq. (18), is the difference between the maintenance cost with a perfect component $k$, and the maintenance cost with the actual component $k$.

Non-coherent systems. The preceding sections are developed on the basis of the assumption that the system is coherent, which of course applies to most systems in practice. However, some extended versions of the Birnbaum importance have been presented for non-coherent systems (e.g. [24]25]). One may follow such approaches to extend the cost-based importance measure for non-coherent systems, this is left as a topic for future research, development of which will greatly benefit from direct application to a real-world non-coherent system to ensure that the specific system features are taken into account.

\section{$5 \quad$ Examples}

We present a numerical example and a small case study to illustrate the basic application of the cost-based importance measure presented in this paper, and to briefly compare it to the Birnbaum importance measure. 


\subsection{A numerical example}

Consider the system shown in Figure 1, for which we will compare cost-based importance measures of different components under different settings.

Using the Birnbaum importance measure, we obtain

$$
\begin{aligned}
& I_{1}^{B}(t)=R_{2}(t)+R_{3}(t)-R_{2}(t) R_{3}(t) \\
& I_{2}^{B}(t)=R_{1}(t)\left(1-R_{3}(t)\right) \\
& I_{3}^{B}(t)=R_{1}(t)\left(1-R_{2}(t)\right)
\end{aligned}
$$

Under Scenario A, the new cost-based importance measure for each component is

$$
\begin{aligned}
I_{1}^{C}(t) & =\frac{c_{s, 1}+c_{1}}{R_{1}(t)} \\
I_{2}^{C}(t) & =\frac{c_{2}}{R_{2}(t)} \\
I_{3}^{C}(t) & =\frac{c_{3}}{R_{3}(t)}
\end{aligned}
$$

Table 2 presents a comparison of the cost-based importance and the Birnbaum importance, assuming that the time interval considered is $(0,4)$ and $c_{s, 1}=$ $c_{s, 2}=c_{s, 3}=100$.

\section{Here: Table 2}

The system in Figure 1 has two minimal cut sets: $U_{1}=\{1\}$ and $U_{2}=\{2,3\}$. Then with Eq. (6), we have

$$
C(t)=\Lambda_{U_{1}}(t)\left(c_{c, 1}+c_{1}\right)+\Lambda_{U_{2,3}}(t)\left(c_{c, 2}+c_{2}+c_{3}\right)
$$


Under Scenario B and with Eqs. (25) and (3), we have

$$
\begin{aligned}
& I_{1}^{C}(t)=\frac{c_{s, 1}+c_{1}}{R_{1}(t)} \\
& I_{2}^{C}(t)=\frac{\left(c_{s, 2}+c_{2}+c_{3}\right)\left(1-R_{3}(t)\right)}{R_{2}(t)+R_{3}(t)-R_{2}(t) R_{3}(t)} \\
& I_{3}^{C}(t)=\frac{\left(c_{s, 3}+c_{2}+c_{3}\right)\left(1-R_{2}(t)\right)}{R_{2}(t)+R_{3}(t)-R_{2}(t) R_{3}(t)}
\end{aligned}
$$

\section{Here: Table 3}

Tables 2 and 3 illustrate how the cost-based importance measure depends on the location of the component in the system, the reliability of the component, the maintenance costs of components upon failure and cost of system failure. Comparison between the cost-based importance measures and the Birnbaum importance measures are also shown in the two tables.

\subsection{A case study}

Andrawus [18] studies the reliability of a $600 \mathrm{~kW}$ wind turbine system, as represented in Fig. 3. Cost data, reliability parameters of each component and cost of system failure are given in Table 4, where the time unit of the scale parameters in the Weibull distributions is hour.

\section{Here: Figure 3}

\section{Here: Table 4}

The Birnbaum importance measure for each component is 


$$
\begin{aligned}
I_{b a}^{B}(t) & =R_{m s}(t) R_{g b}(t) R_{g}(t)\left(1-R_{b b}(t)\right) \\
I_{b b}^{B}(t) & =R_{m s}(t) R_{g b}(t) R_{g}(t)\left(1-R_{b a}(t)\right) \\
I_{m s}^{B}(t) & =R_{g b}(t) R_{g}(t)\left(R_{b a}+R_{b b}-R_{b a}(t) R_{b b}(t)\right) \\
I_{g b}^{B}(t) & =R_{m s}(t) R_{g}(t)\left(R_{b a}+R_{b b}-R_{b a}(t) R_{b b}(t)\right) \\
I_{g}^{B}(t) & =R_{m s}(t) R_{g b}(t)\left(R_{b a}+R_{b b}-R_{b a}(t) R_{b b}(t)\right)
\end{aligned}
$$

Based on Eqs. 29 - 33) and Table 4, the ordering of the Birnbaum importance measures of the components is given by

$$
I_{m s}^{B}(t)>I_{g}^{B}(t)>I_{g b}^{B}(t)>I_{b a}^{B}(t)=I_{b b}^{B}(t)
$$

As [18] explained, the reliability, availability and maintainability of the wind turbine are assessed over a period of 4 years; taking into account the costs and availability of maintenance crew and spares holding. The ' 4 years' is a short term economic analysis period required by the collaborating wind farm operator. We therefore consider the cost-based importance measure assuming that the time interval under investigation is $(0,4)$ years.

Under Scenario A, we have

$$
\begin{aligned}
I_{b a}^{C}(t) & =\frac{c_{b a}}{R_{b a}(t)} \\
I_{b b}^{C}(t) & =\frac{c_{b b}}{R_{b b}(t)} \\
I_{m s}^{C}(t) & =\frac{c_{s, m s}+c_{m s}}{R_{m s}(t)} \\
I_{g b}^{C}(t) & =\frac{c_{s, g b}+c_{g b}}{R_{g b}(t)} \\
I_{g}^{C}(t) & =\frac{c_{s, g}+c_{g}}{R_{g}(t)}
\end{aligned}
$$


Based on Eqs. (33)-(37) and Table 4 , the ordering of the cost-based importance measures of the components is given by

$$
I_{g b}^{C}(t)>I_{g}^{C}(t)>I_{m s}^{C}(t)>I_{b a}^{C}(t)=I_{b b}^{C}(t)
$$

Inequality (40) shows a different ordering from Inequality (32), that is, the ordering according to the cost-based importance measure differs from that according to the Birnbaum importance measure. Of course, the importance ordering derived from the cost-based importance reflects the different costs involved, which is probably more useful in practice. The possibility to do so, in a way that generalizes Birnbaum importance, will be attractive in many applications and can provide important insights. For example, in this case study we see that improving the reliability of the gear box can result in the largest cost reduction.

\section{Conclusions}

This paper introduced a new cost-based importance measure, for repairable systems. We derived the cost-based importance for two maintenance scenarios A and B. For Scenario A, we considered a general case where components in the system can have any lifetime distributions, and derived the cost-based importance for each component. For Scenario B, we discussed the challenges to obtain cost-based importance when components in the system have general lifetime distributions, and we derived the cost-based importance for each component only when they have exponential lifetime distributions for a special case.

The novelty of this paper lies in the fact that it proposed a new importance measure, which takes consideration of costs of repairing components and cost of repairing the system.

The data example shows that the ordering of the cost-based importance can be 
different from the Birnbaum importance and it relates not only to component reliability and location but also cost on maintenance. This indicates that the cost-based importance can be very useful as it takes different types of cost into consideration.

\section{Acknowledgements}

We are grateful to the reviewers for their helpful comments that lead an improvement on the paper.

\section{References}

[1] Z. Birnbaum, On the importance of different components in a multicomponent system, in Multivariate analysis II, Academic Press, New York, 1969, pp. 581592.

[2] R. E. Barlow, F. Proschan, Importance of system components and fault tree events, Stochastic Processes and their Applications 3 (2) (1975) 153-173.

[3] J. B. Fussell, How to hand-calculate system reliability and safety characteristics., IEEE Transactions on Reliability R-24 (3) (1975) 169-174.

[4] B. Natvig, A suggestion of a new measure of importance of system components, Stochastic Processes and their Applications 9 (3) (1979) 319-330.

[5] W. S. Griffith, Multistate reliability models, Journal of Applied Probability 17 (1980) 735-744.

[6] P. J. Boland, F. Proschan, Y. L. Tong, Optimal arrangement of components via pairwise rearrangements, Naval Research Logistic Quarterly 36 (1989) 455-463.

[7] S. Wu, L. Chan, Performance utility-analysis of multi-state systems, IEEE Transactions on Reliability 52 (1) (2003) 14-21.

[8] S. Wu, Joint importance of multistate systems, Computers and Industrial Engineering 49 (1) (2005) 63-75. 
[9] X. Gao, L. Cui, J. Li, Analysis for joint importance of components in a coherent system, European Journal of Operational Research 182 (1) (2007) 282-299.

[10] T. Aven, T. Nokland, On the use of uncertainty importance measures in reliability and risk analysis, Reliability Engineering and System Safety 95 (2) (2010) 127-133.

[11] E. Borgonovo, The reliability importance of components and prime implicants in coherent and non-coherent systems including total-order interactions, European Journal of Operational Research 204 (3) (2010) 485-495.

[12] P. Do Van, A. Barros, C. Brenguer, From differential to difference importance measures for markov reliability models, European Journal of Operational Research 204 (3) (2010) 513-521.

[13] S. Contini, V. Matuzas, New methods to determine the importance measures of initiating and enabling events in fault tree analysis, Reliability Engineering and System Safety 96 (7) (2011) 775-784.

[14] S. Si, H. Dui, X. Zhao, S. Zhang, S. Sun, Integrated importance measure of component states based on loss of system performance, IEEE Transactions on Reliability 61 (1) (2012) 192-202.

[15] H. Peng, D. W. Coit, Q. Feng, Component reliability criticality or importance measures for systems with degrading components, IEEE Transactions on Reliability 61 (1) (2012) 4-12.

[16] Y. Xiang, G. Levitin, Combined m-consecutive and k-out-of-n sliding window systems, European Journal of Operational Research 219 (1) (2012) 105-113.

[17] M. Rausand, A. Hoyland, System Reliability Theory: Models, Statistical Methods, and Applications, John Wiley \& Sons, New Jersey, 2004.

[18] J. A. Andrawus, Maintenance optimization for wind turbines, Ph.D. thesis, Robert Gordon University (2008).

[19] P. Coolen-Schrijner, F. P. A. Coolen, On optimality criteria for age replacement, Proceedings of the Institution of Mechanical Engineers, Part O: Journal of Risk and Reliability 220 (2006) 21-28. 
[20] P. Coolen-Schrijner, F. P. A. Coolen, Nonparametric adaptive age replacement with a one-cycle criterion, Reliability Engineering and System Safety 92 (1) (2007) 74-84.

[21] S. Kaplan, B. J. Garrick, On the quantitative definition of risk, Risk Analysis 1 (1) (1981) 11-27.

[22] A. P. Sage, E. B. White, Methodologies for risk and hazard assessment: A survey and status report, IEEE Transactions on Systems, Man and Cybernetics 10 (8) (1980) 425-446.

[23] B. Natvig, A. B. Huseby, M. O. Reistadbakk, Measures of component importance in repairable multistate systems - a numerical study, Reliability Engineering and System Safety 96 (12) (2011) 1680-1690.

[24] P. S. Jackson, On the s-importance of elements and prime implicants of noncoherent systems, IEEE Transactions on Reliability R-32 (1) (1983) 21-25.

[25] J. D. Andrews, S. Beeson, Birnbaum's measure of component importance for noncoherent systems, IEEE Transactions on Reliability 52 (2) (2003) 213-219. 
Table 1

Notation

$c_{k} \quad$ cost per failure of component $k$

$c_{s, k} \quad$ expected cost per system failure caused by the failure to component $k$

$c_{c, i}$ expected cost per system failure caused by the failure of minimal cut set $i$, where $i=1, \ldots, n_{0}$

$C(t)$ expected total cost of maintaining the system within time $(0, t)$

$C_{k}(t) \quad$ expected total cost of maintaining component $k$ within time $(0, t)$

$C_{s, k}(t)$ expected total cost incurred due to system failure within time $(0, t)$, caused by the failure of component $k$

$m_{0}$ total number of different minimal cut sets, each of which contains only one component

$n$ number of components in the system

$n_{0} \quad$ total number of different minimal cut sets in the system

$R_{k}(t) \quad$ reliability function of component $k$

$R_{s}(t) \quad$ reliability of the system

$U_{i_{1}, \ldots, i_{m_{i}}}$ the $i$-th minimal cut set, which contains components $i_{1}, i_{2}, \ldots, i_{m_{i}}$, where $i=$ $1,2, \ldots, n_{0}$

$\Lambda_{k}(t) \quad \Lambda_{k}(t)=\int_{0}^{t} \lambda_{k}(x) d x$, where $\lambda_{k}(t)$ is the failure intensity function associated with $F_{k}(t)$, where $F_{k}(t)=1-R_{k}(t)$

$\Lambda_{s}(t) \quad \Lambda_{s}(t)=\int_{0}^{t} \lambda_{s}(x) d x$, where $\lambda_{s}(t)$ is the failure intensity function associated with $F_{s}(t)$ and $F_{s}(t)=1-R_{s}(t)$

$\Lambda_{U_{i_{1}, \ldots, i_{m_{i}}}}(t) \quad \int_{0}^{t} \lambda_{U_{i_{1}, \ldots, i_{m_{i}}}}(x) d x$, where $\lambda_{U_{i_{1}, \ldots, i_{m_{i}}}}(t)$ is the failure intensity function associated with $F_{U_{i_{1}, \ldots, i_{m}}}(t)$ 
Table 2

Comparison between the Birnbaum importance and the cost-based importance under Scenario A.

\begin{tabular}{|c|c|c|c|}
\hline Reliabilities & Costs & Birnbaum importance & Cost-based importance \\
\hline$R_{1}(t)=R_{2}(t)=R_{3}(t)=e^{-0.1 t^{2}}$ & $\begin{array}{l}c_{3}=c_{4}=c_{5}=20 \\
c_{3}=20 ; c_{4}=c_{5}>120 \\
c_{3}=20 ; c_{4}>120 ; c_{5}>c_{4}\end{array}$ & $\begin{array}{l}I_{1}^{B}(t)>I_{2}^{B}(t)=I_{3}^{B}(t) \\
I_{1}^{B}(t)>I_{2}^{B}(t)=I_{3}^{B}(t) \\
I_{1}^{B}(t)>I_{2}^{B}(t)=I_{3}^{B}(t)\end{array}$ & $\begin{array}{l}I_{1}^{C}(t)>I_{2}^{C}(t)=I_{3}^{C}(t) \\
I_{1}^{C}(t)<I_{2}^{C}(t)=I_{3}^{C}(t) \\
I_{1}^{C}(t)<I_{2}^{C}(t)<I_{3}^{C}(t)\end{array}$ \\
\hline $\begin{array}{l}R_{1}(t)=e^{-0.1 t^{2}} ; R_{2}(t)=e^{-0.2 t^{2}} ; \\
R_{3}(t)=e^{-0.3 t^{2}}\end{array}$ & $\begin{array}{l}c_{3}=c_{4}=c_{5}=20 \\
c_{3}=500 ; c_{4}=c_{5}=20 \\
c_{3}=500 ; c_{4}=100 ; c_{5}=20\end{array}$ & $\begin{array}{l}I_{1}^{B}(t)<I_{2}^{B}(t)<I_{3}^{B}(t) \\
I_{1}^{B}(t)<I_{2}^{B}(t)<I_{3}^{B}(t) \\
I_{1}^{B}(t)<I_{2}^{B}(t)<I_{3}^{B}(t)\end{array}$ & $\begin{array}{l}I_{2}^{C}(t)<I_{1}^{C}(t)<I_{3}^{C}(t) \\
I_{1}^{C}(t)>I_{3}^{C}(t)>I_{2}^{C}(t) \\
I_{1}^{C}(t)>I_{2}^{C}(t)>I_{3}^{C}(t)\end{array}$ \\
\hline
\end{tabular}

Table 3

Comparison between the Birnbaum importance and the cost-based importance, under Scenario B, given that a time interval $(0,4)$ and $c_{s}=100$.

\begin{tabular}{l|lrr}
\hline \hline Reliabilities & Costs & Birnbaum importance & Cost-based importance \\
\hline & $c_{3}=c_{4}=c_{5}=20$ & $I_{1}^{B}(t)>I_{2}^{B}(t)=I_{3}^{B}(t)$ & $I_{1}^{C}(t)>I_{2}^{C}(t)=I_{3}^{C}(t)$ \\
$R_{1}(t)=R_{2}(t)=R_{3}(t)=e^{-0.1 t}$ & $c_{3}=10 ; c_{4}=c_{5} \geq 175$ & $I_{1}^{B}(t)>I_{2}^{B}(t)=I_{3}^{B}(t)$ & $I_{1}^{C}(t)<I_{2}^{C}(t)=I_{3}^{C}(t)$ \\
& for any $c_{4}$ and $c_{5}$ & $I_{1}^{B}(t)>I_{2}^{B}(t)=I_{3}^{B}(t)$ & $I_{2}^{C}(t)=I_{3}^{C}(t)$ \\
\hline & $c_{3}=c_{4}=c_{5}=20$ & $I_{1}^{B}(t)<I_{2}^{B}(t)<I_{3}^{B}(t)$ & $I_{1}^{C}(t)>I_{3}^{C}(t)>I_{2}^{C}(t)$ \\
$R_{1}(t)=e^{-0.1 t} ; R_{2}(t)=e^{-0.2 t} ;$ & $c_{3}=10 ; c_{4}=c_{5}=45$ & $I_{1}^{B}(t)<I_{2}^{B}(t)<I_{3}^{B}(t)$ & $I_{3}^{C}(t)>I_{2}^{C}(t)>I_{1}^{C}(t)$ \\
$R_{3}(t)=e^{-0.3 t}$ & for any $c_{4}$ and $c_{5}$ & $I_{1}^{B}(t)<I_{2}^{B}(t)<I_{3}^{B}(t)$ & $I_{3}^{C}(t)>I_{2}^{C}(t)$ \\
\hline \hline
\end{tabular}

Table 4

Cost data and reliability parameters [18].

\begin{tabular}{l|lll}
\hline \hline Component name & $\begin{array}{l}\text { Expected cost of per } \\
\text { component failure }(£)\end{array}$ & $\begin{array}{l}\text { Expected cost of system failure } \\
\text { due to a component failure }(£)\end{array}$ & $\begin{array}{l}\text { Reliability } \\
\text { (Weibull parameters } \\
\text { distributions: } \\
\text { the unit of the scale } \\
\text { parameters is day) }\end{array}$ \\
\hline Bearing A & $c_{b a}=42204$ & $c_{s, b a}=22375$ & $R_{b a}(t)=e^{-\left(\frac{t}{3835}\right)^{1.09}}$ \\
Bearing B & $c_{b b}=42204$ & $c_{s, b b}=22375$ & $R_{b b}(t)=e^{-\left(\frac{t}{3835}\right)^{1.09}}$ \\
Main shaft & $c_{m s}=27266$ & $c_{s, m s}=29114$ & $R_{m s}(t)=e^{-\left(\frac{t}{6389}\right)^{1.43}}$ \\
Gear box & $c_{g b}=65387$ & $c_{s, g b}=78468$ & $R_{g b}(t)=e^{-\left(\frac{t}{29051}\right)^{1.05}}$ \\
Generator & $c_{g}=49482$ & $c_{s, g}=35964$ & $R_{g}(t)=e^{-\left(\frac{t}{17541}\right)^{1.11}}$ \\
\hline \hline
\end{tabular}




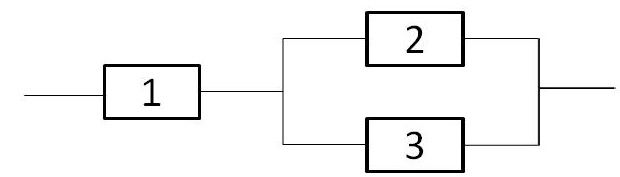

Fig. 1. System Example 1.

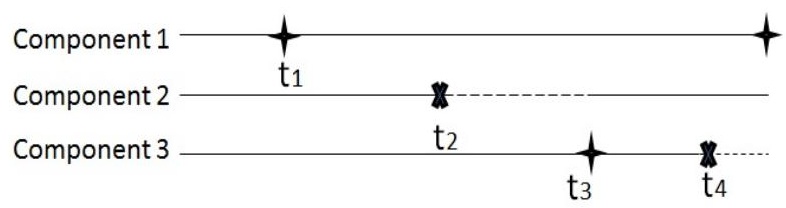

-: Operating $\quad---$ : Waiting for repair

: Component fails but the system is still operating

t: Component fails and the system fails

Fig. 2. A typical case of Scenario B.

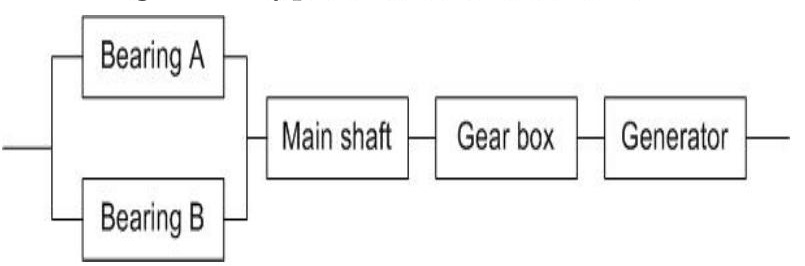

Fig. 3. A 600kW Wind Turbine (Example 2). 


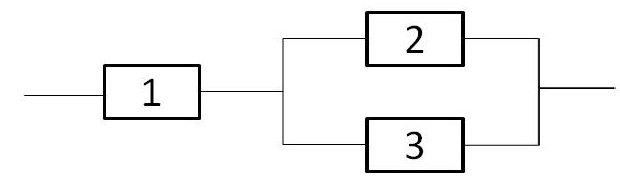

Fig. 1. System Example 1.

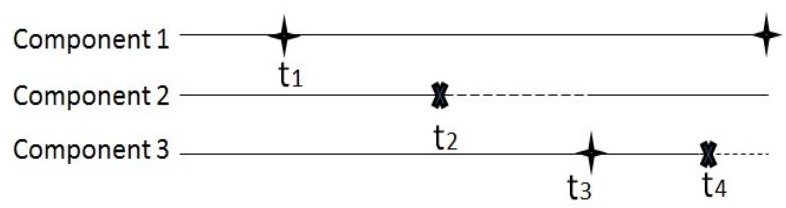

-: Operating $\quad---$ : Waiting for repair

: Component fails but the system is still operating

t: Component fails and the system fails

Fig. 2. A typical case of Scenario B.

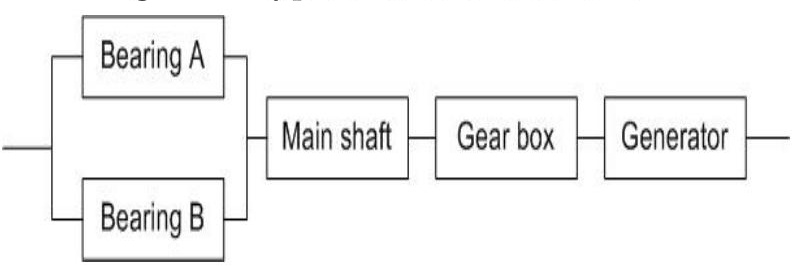

Fig. 3. A 600kW Wind Turbine (Example 2). 